\title{
Leveraging public health nurses for disaster risk communication in Fukushima City: a qualitative analysis of nurses' written records of parenting counseling and peer discussions
}

\author{
Aya Goto ${ }^{1,2^{*}}$, Rima E Rudd ${ }^{3}$, Alden Y Lai ${ }^{4}$, Kazuki Yoshida ${ }^{1}$, Yuu Suzuki ${ }^{1}$, Donald D Halstead ${ }^{5}$,
} Hiromi Yoshida-Komiya ${ }^{6}$ and Michael R Reich ${ }^{7}$

\begin{abstract}
Background: Local public health nurses (PHNs) have been recognized as the main health service providers in communities in Japan. The Fukushima nuclear disaster in 2011 has, however, created a major challenge for them in responding to mothers' concerns. This was in part due to difficulties in assessing, understanding and communicating health risks on low-dose radiation exposure. In order to guide the development of risk communication plans, this study sought to investigate mothers' primary concerns and possible solutions perceived by a core healthcare profession like the PHNs.

Methods: A total of 150 records from parenting counseling sessions conducted between PHNs and mothers who have attended mandatory 18-month health checkups for their children at the Fukushima City Health and Welfare Center in 2010, 2011 (year of disaster) and 2012 were examined. Discussion notes of three peer discussions among PHNs organized in response to the nuclear disaster in 2012 and 2013 were also analyzed. All transcribed data were first subjected to text mining to list the words according to their frequencies and inter-relationships. The Steps Coding and Theorization method was then undertaken as a framework for qualitative analysis.
\end{abstract}

Results: PHNs noted mothers to have considerable needs for information on radiation risks as they impact on decisions related to relocations, concerns for child safety, and experiences with interpersonal conflicts within the family owing to differing risk perceptions. PHNs identified themselves as the information channels in the community, recommended the building of their risk communication capacities to support residents in making well-informed decisions, and advocated for self-measurement of radiation levels to increase residents' sense of control. PHNs also suggested a more standardized form of information dissemination and an expansion of community-based counseling services.

Conclusions: Inadequate risk communication on radiation in the Fukushima nuclear incident has resulted in multiple repercussions for mothers in the community. Empowerment of local residents to assume more active roles in the understanding of their environment, increasing PHNs' capacity in communication, and an expansion of health services such as counseling will together better address risk communication challenges in post-disaster recovery efforts.

Keywords: Public health nurses, Risk communication, Parenting, Radiation, Health communication, Fukushima nuclear accident, Japan

\footnotetext{
* Correspondence: agoto@fmu.ac.jp

1 Department of Public Health, Fukushima Medical University School of

Medicine, Hikarigaoka 1, Fukushima-City, Fukushima 960-1295, Japan

${ }^{2}$ Takemi Program in International Health, Harvard School of Public Health

(at time of manuscript writing), 665 Huntington Avenue, Boston, MA 02115, USA

Full list of author information is available at the end of the article
} 


\section{Background}

Experience from past nuclear accidents shows that poor risk communication increases uncertainty and panic among the public [1], which has also been observed after the Fukushima nuclear disaster that occurred on March 11, 2011 following the Great East Japan Earthquake. The central government of Japan failed to inform the municipal governments of the occurrence and severity of the incident in a timely manner, leading to chaotic migrations among residents, and eventually causing excess mortality among vulnerable populations such as the institutionalized elderly [2,3]. Mothers of young children are among the most-affected in the Fukushima nuclear incident, as inconsistent information about radiation levels in breast milk posted by two different professional organizations (the Japanese Society of Obstetrics and Gynecology and the Japan Radiological Society) had further created high levels of confusion in terms of maintaining safety for their children [4].

This confusion around risk-related information continued into 2013 [5]. Two years after the disaster, the World Health Organization reported that in the two most affected locations of the Fukushima prefecture, the lifetime risk of thyroid cancer among girls exposed as infants had increased by up to $70 \%$. However, the baseline risk is low and even a large relative increase only represents an absolute increase of as low as 0.005 in terms of life-time cumulated probability of developing the cancer [6]. The concept of risk is difficult to understand, even among health professionals, and its comprehension is even more complicated when a relative risk is high in spite of a low absolute risk [7]. This leads to major challenges for people, especially for families with small children in deciding on how to address the risks they are exposed to. In this case, Fukushima City is located about $70 \mathrm{~km}$ from the nuclear power plant, and the estimated additional lifetime risk of thyroid cancer is approximately half of that in the two locations mentioned above [6]. Nevertheless, the population of children under 5 years old in the city had declined by nearly $15 \%$ during the two years following the disaster.

Although the importance of empowering local residents via proper risk communication to allow them to make autonomous decisions in post-disaster recovery processes is recognized and recommended in Japan at the national level [2], no guidelines have been developed on how to plan or implement this at the community level. This has led to confusion among public health nurses (PHNs), who in Japan work in a public sector, provide a wide range of community health services, and are often the first point of contact with residents. The Japanese government has recognized the pivotal role they play, as PHNs are tasked with community assessment, health planning, service provision, networking and service evaluation at the municipal level. However, PHNs have voiced concerns about their safety and their insufficient level of knowledge to provide adequate services in the aftermath of the nuclear disaster [8]. In the year before the disaster, researchers from the National Mental Health Center in Japan had already revised the disaster mental health guidelines that were endorsed by mental health professionals. However, an agreement was not reached in defining the roles of PHNs in a disaster setting [9].

To develop proper maternal and child health strategies that can be adopted to support residents facing anxiety about radiation, regular meetings were held in Fukushima City in 2011 between a team of public health researchers from Fukushima Medical University (organized by the first author) and city PHNs in reaction to the disaster [10]. These meetings were expanded in the subsequent year to include three training workshops, aiming to provide PHNs with knowledge on the health effects of radiation exposure and create opportunities for information sharing among peer nurses. In addition, there were collaborations with PHNs to derive additional insight from their routine work. This included the analyses of child health checkup records written by PHNs, with particular attention to information from parenting counseling sessions conducted between PHNs and mothers, as they are one of the most affected population groups in the disaster. Mothers in Japan are required to report their pregnancies to a municipal office, and municipalities are mandated to provide health checkups for 18 and 36 months old children. PHNs are the main service providers of such checkups, as they assess children's physical health while providing mothers with parenting counseling. As this community model of needs assessment and strategy planning by PHNs with technical support from a local university amidst an emergency situation is rare, both in the Fukushima context and scientific literature on disaster recovery, the understanding derived from this model is instrumental in alleviating existing post-disaster challenges in Japan and in future disaster occurrences.

The concerns of disaster-affected residents have to be carefully examined so that communication needs and strategies for improving risk communication in a community setting following a disaster can be appropriately delineated. This study focuses on mothers and PHNs (given their role in the Japanese community health setting) following the nuclear incident in Fukushima. Launching new systematic surveys in a post-disaster situation was not feasible. Concern with not overburdening either community members or professionals, information from existing healthcare services and disaster recovery efforts (i.e., mandated child health checkups and the aforementioned community model between the University and 
city PHNs) were capitalized upon in Fukushima City to undertake this line of academic inquiry.

In this study, a qualitative approach has been adopted to look at existing records of parenting counseling to examine the residents' concerns after the disaster with the nuclear incident, and PHNs' peer discussions to delineate possible strategies that can be taken to alleviate these concerns (see Figure 1). The overarching aim is to elicit insights into steps that can be undertaken for risk communication strategies during disaster recovery efforts. Although health planning processes of PHNs have been investigated by previous research [11], little is known about the challenges they face in a crisis setting. The primary advantage of our study design is the possibility of documenting concerns within the community before and after the disaster, as a significant limitation in disaster studies is often the lack of baseline data [12]. Moreover, using two different sources of qualitative data have allowed us to explore issues from varying perspectives. This research will inform current and future efforts in disaster recovery, especially in terms of facilitating appropriate communication on topics of major concern in situations of disasters and crises.

\section{Methods}

This was a qualitative analysis study utilizing two available data collected at the Fukushima City Health and Welfare Center and applying two analytical methods that are highly practical.

\section{Data collection}

Data was drawn from parenting counseling records and from peer discussions among PHNs.

\section{Parenting counseling records}

Both quantitative and qualitative data on parenting counseling were collected from a series of crosssectional studies consisting of mothers who attended their children's 18-month health checkups at the Fukushima City Health and Welfare Center between

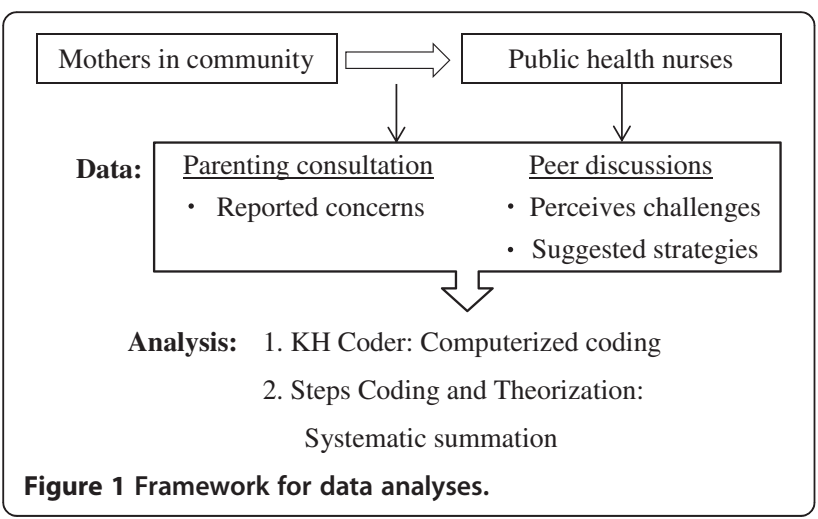

April to May of 2010, 2011 and 2012. In Japan, mothers are required to report their pregnancies to a municipal office, and the municipal offices are in turn mandated to provide child health checkups. The attendance rate of the 18-month health checkups in Fukushima City for these three years was over 90\%. It was one month after the disaster (i.e., April 2011) when regular health services including children's health checkups resumed in the city. Based on sample size calculation for analyses of quantitative data (to detect expected associations), the study period was specified to be from April to May. The number of mothers enrolled in the checkups was 156 in 2010, 218 in 2011, and 131 in 2012. For each year, PHNs' records from parenting counseling were scanned from the child health checkup files for the first consecutive 50 attendants and then transcribed into a database. Based on our previous pilot study, it was known that approximately $30 \%$ of mothers would voice concerns about effects of radiation in parenting counseling after the disaster [10]. The amount of qualitative data to be derived from each counseling session was also known. Coupling these insights, it was discerned with consensus among the authors involved in data collection that a sample size of 50 records of parenting counseling sessions per year will generate sufficient data for analyses.

2. Nurses' peer discussions

We collected another set of data from PHNs attending three training workshops conducted in July and October 2012, and January 2013. These workshops were co-organized by the Gender-Specific Medicine Center and Department of Public Health of Fukushima Medical University School of Medicine, aiming to provide professional training and information sharing opportunities for PHNs in Fukushima City. The number of attendants was 20, 29 and 25 in each workshop, respectively. There were 39 PHNs working at the Fukushima City Health and Welfare Center at the time of study, and all except those assigned to service provision duties when the workshops took place attended. Each workshop consisted of a one-hour lecture by a university physician, followed by an hour of group discussion. The topics for each lecture were, in order of occurrence, women's health, radiation, and thyroid cancer. In the group discussion, we handed each PHN three note sheets and asked them to write down their reflections after the lectures. All collected note sheets were then systematically transcribed into our database.

\section{Analysis plan}

Both datasets were subjected to identical analysis methods (refer to Figure 1) - transcribed data were first analyzed by 
text mining using the $\mathrm{KH}$ Coder, a software program developed by K. Higuchi at Ritsumeikan University in Japan [13]. This text mining is a computerized process of extracting information from collected information, and has increasingly received attention among researchers as a way to improve the consistency of qualitative analysis, especially during the coding stage [14]. The $\mathrm{KH}$ coder has recently been used to analyze medical articles in academic journals and newspapers in Japanese [15]. The program segments sentences, lists frequently used words, and develops a hierarchical analysis diagram showing relationships among words with their corresponding frequencies.

We then focused on the top 20\% of the words that were most frequently listed, and reviewed sentences that included these words to examine the full context in which the words were used. By referring to a diagram generated by the $\mathrm{KH}$ coder, we then categorized the words into major topics, eliminating common words that appeared in sentences across the different topics after categorization (e.g., "mother" and "think" in the parenting counseling data). We then calculated the proportion of cases that included each topic. A "case" refers to a mother in the parenting counseling data, or a note sheet from the PHNs' workshop data. Since the $\mathrm{KH}$ Coder calculates word frequencies without analyzing the full context, some misclassifications were observed and manually eliminated when calculating this proportion of cases in each topic. This analysis procedure was repeated twice by the first author with a one-month interval in between to ensure consistency.

Second, cases related to specific topics of our interest (such as "disaster-related issues" among the parenting counseling data, and "action plans" among the workshop data) were analyzed by Steps Coding and Theorization (SCAT) [16]. SCAT was developed as an accessible qualitative data analysis method by $T$. Otani from Nagoya University in Japan. This method is appropriate for small-scale qualitative studies with a limited amount of qualitative data including answers to open-ended questions in surveys, and has been used by researchers in the fields of medical education and palliative care [17]. The analysis consists of two steps - first decontextualization to generate themes from sentences, followed by theorization to construct theories summarizing collected information. In the first decontextualization process, we extracted key words from original sentences, rephrased them by using professional terms, created themes, and then labeled each case. In the second recontexualization process, we developed a storyline from the emerging themes. The first and third authors independently performed the SCAT analysis of data extracted from each workshop, and the first author then compared and combined the results, with consensus achieved with the third author subsequently.
Compared with the classic grounded theory for qualitative analysis, which often requires a large amount of text data, the $\mathrm{KH}$ coder enhances consistency and reproducibility especially in data coding [14], and SCAT further enables systematic analysis of a small amount of text data [16]. We thus applied $\mathrm{KH}$ coder first to extract categories and then SCAT to deepen our interpretation of the categories that are most pertinent to our study's objectives. One of this study's aims is to test the usefulness of these methods in a community health practice setting.

\section{Ethical considerations}

This study was conducted in collaboration with the Fukushima City Health and Welfare Center. All data were copied anonymously without any identifiers of families and PHNs, and conducted in accordance with Ethical Guidelines for Epidemiological Research issued by the Japanese Ministry of Education, Culture, Sports, Science and Technology, and the Japanese Ministry of Health, Labour and Welfare. Data collection on parenting counseling was submitted to the ethical review boards at the Fukushima Medical University and the city office, and reviews were waived. The data analysis plan of the PHNs' group discussions was submitted to an IRB Review Specialist at the Harvard School of Public Health, but a review was also waived as this was categorized as a quality improvement study.

\section{Results}

1. Parenting counseling

Figure 2 shows trends found in parenting counseling topics categorized using the $\mathrm{KH}$ Coder. The proportions of "child lifestyle" and "communication with a child" decreased while those of "child medical issues", "disaster-related issues," "parental concerns," and "support network" increased. The words included in each topic are listed in Table 1.

The cases that included words in the "disasterrelated issues" topic - the records of 13 mothers in 2011 and 9 mothers in 2012-were analyzed by SCAT. In 2011, these mothers experienced relocation and changes in daily family routines. Mothers were worried about children's radiation exposure from playing outside, and needed more information about radiation. These changes in daily life and anxiety toward radiation had negative psychological impacts. In 2012, mothers continued to relocate and voiced concerns about letting their children play outside. They started to ask questions about technical issues, including radiation measurement procedures, and raised 


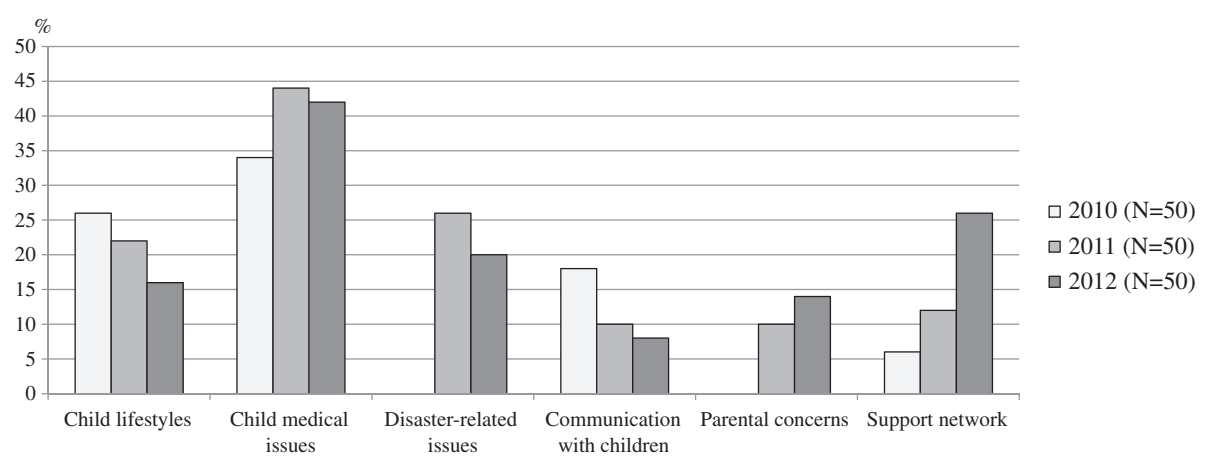

Figure 2 Proportions of major topics: public health nurses' records of parenting counseling. Number of women (denominator) is 50 in each year.

concerns about differences in risk perception toward radiation with their spouses. PHNs recorded:

"I was asked] What does it mean to measure a parent's exposure level? Another city introduced a machine that a child can get into [and be measured directly]."

"The mother is worried about radiation and cannot let [child] play outside. [She worries about] her child licking the sand from her hands after falling down. The father says it is alright to play outside for a short time."

2. Nurses' peer discussions In the $\mathrm{KH}$ Coder analysis of the workshop data, we created two topics, "action plans" and "learned knowledge." Since most of the notes included words categorized as "learned knowledge," Figure 3 focuses on the proportion of notes including words categorized as "action plans." We found that $18 \%$ of notes in the first workshop mentioned action plans, but this figure increased to $47 \%$ in the second workshop, and was $43 \%$ in the third workshop. Among words related to "action plans" as listed in
Table 2, words (used as a noun or verb) that appeared across all three workshops were "inform" and "knowledge."

The cases that included words in the "action plans" topic-the records of 17, 36, and 35 note sheets in three workshops, respectively-were analyzed by SCAT. PHNs identified their role as an information channel, and emphasized the importance of supporting residents to make autonomous decisions based on credible evidence. More specifically, they recommended measuring radiation levels as a useful tool for residents to have a clear understanding of their own environment. PHNs wrote:

"It is important for mothers to think by themselves based on accurate information. Public health nurses are their close advisors."

"By measuring and calculating your own exposure level, anxiety and worries can be reduced."

"[Use] radiation dosimeters. Calculate correctly."

PHNs suggested the need to relay messages in a clearer manner and to have better community-based

Table 1 Words included in major topics: Public health nurses' records of parenting counseling

\begin{tabular}{ll}
\hline Major topics & Words in alphabetical order \\
\hline Child lifestyles & $\begin{array}{l}\text { Banana, breast milk, crying at night, drink, eat, feed, irregular, meal, milk (two words), morning, nap, night, sleep } \\
\text { (two words), vegetable }\end{array}$ \\
Child medical issues & $\begin{array}{l}\text { Apply, bleed, brushing, curved, dry, ear, ear infection, eyes, feet, front teeth, height, immunization, legs, live (vaccine), } \\
\text { medicine, nails, occlusion of teeth, pediatrics, prescription, prevention, polio, receive, run, skin, teeth, tooth decay, treat, } \\
\text { vaccination, weight, words }\end{array}$ \\
Disaster-related issues & $\begin{array}{l}\text { Daily life, earthquake, evacuation, Fukushima, nuclear, outside, play, radiation } \\
\text { Communication with children }\end{array}$ \\
Children, cry, dependent, dislike, elder child, hold \\
Parenting network & Job, mind, overwhelmed, upset \\
\hline
\end{tabular}




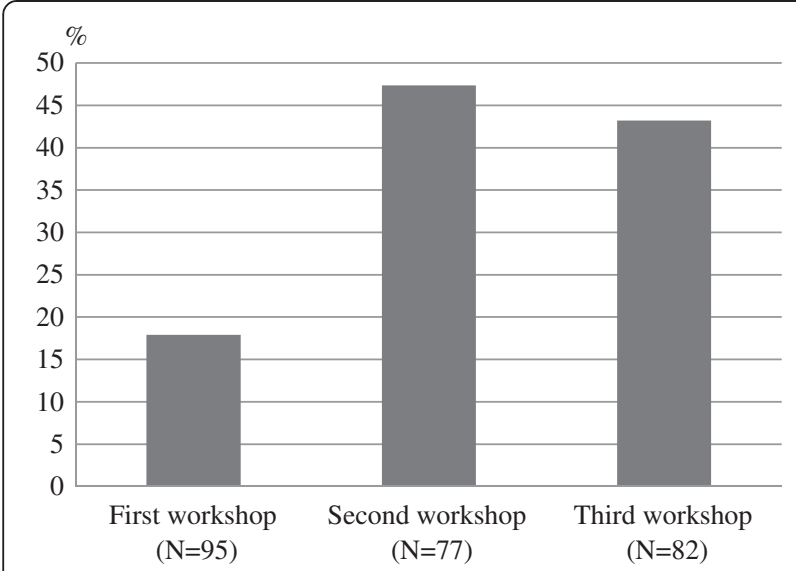

Figure 3 Proportions of discussion notes including "action plans" words: public health nurses' group discussions in workshops. Denominator is the number of discussion notes in each workshop.

counseling service that addresses multiple but related concerns regarding parenting in the context of a disaster like Fukushima:

"Residents demand that we talk to them as the same disaster-affected residents. We must tell them what we think about what we have learned, not just what the government says."

"It is important for public health nurses to learn and share knowledge."

"[Mothers] need a place where they can ask every little question about their daily lives."

In particular, there were strong concerns among PHNs that the purpose and results of thyroid cancer screening were difficult to understand, and that clearer, standardized explanations were needed. They further recognized that the matter was not only about improving access to information, but about providing a more careful follow-up system after the screening, taking its psychological impact into account:
"I understood how to read the screening results, but I am not confident in explaining them to residents." "[Once a thyroid cancer screening is introduced,] we will find cancer cases. It will look like the cancer is increasing. It becomes a problem of how to respond to people's anxiety."

\section{Discussion}

Given the uncertainty caused by inconsistent scientific information around radiation, the analyses of PHNs' interactions with mothers and with their own peers have yielded insight into the challenges and corresponding strategies that PHNs face and can adopt for better communication of risks. This study provides communitybased insights on the needs related to health communication and on the ways to improve risk communication following the Fukushima nuclear accident through the perspectives of PHNs, who live and work in, and interact regularly with the local community. This is the first study to delineate such information through a combination of data from PHNs and their interactions with mothers, and is thought to be instrumental to our general knowledge in disaster recovery interventions despite the context to be specific to the Fukushima Nuclear incident.

\section{Mothers' concerns}

Many disaster studies lack baseline data [12]; in contrast, our review of records from routine services of parenting counseling provides this critical baseline, and enabled us to document the changing trends of community concerns before and after the disaster. Many of the post disaster concerns from Fukushima are similar to those documented in other disaster settings $[18,19]$. The PHNs' records of mothers' reactions after the disaster were similar to those observed after the Chernobyl accident; parental anxiety at a subclinical level is expected according to previous research [18], and the mothers in our study have also raised similar concerns in the year of the Fukushima incident and in the following year. Another observation from the Chernobyl incident was the tendency for mothers to report somatic symptoms of their children despite no abnormalities in medical assessments [19], and our study

Table 2 Words included in major topics: Public health nurses' group discussions in workshops

\begin{tabular}{ll}
\hline Workshops (Lecture topics) & Words categorized as "action plans" in alphabetical order \\
\hline First workshop (Women's health) & Educate, important, inform, knowledge, necessary, promote \\
Second workshop (Radiation) & $\begin{array}{l}\text { Calculate, close, confirm, correct, equip, execute, important, inform, information, knowledge, learn, mothers, } \\
\text { necessary, play, prevention, public health nurses, understand }\end{array}$ \\
Third workshop (Thyroid cancer) & $\begin{array}{l}\text { Adults, anxiety, children, correct, difficult, equip, explain, future, health, important, inform, information, issue, } \\
\text { knowledge, manage, necessary, purpose, radiation, results, risk, safe, situation, think, worry }\end{array}$ \\
\hline
\end{tabular}


has likewise observed an increase in consultations on children's medical issues during health checkups in 2011.

Concerns about their children's health further escalated, as evident from statements by mothers attending consultations in 2012 to question PHNs about technical information related to radiation and thyroid screening. Additional knowledge on radiation and its related health risks in PHNs is clearly warranted. Another finding from our study was how these mothers were also voicing conflicts of risk perceptions with their spouses. This resonates with findings from a recent study looking into risk communication after the Fukushima incident, which has shown a distinct higher proportion of mothers in Fukushima to have stronger levels of anxiety as opposed to fathers [20]. It is well known that risk perception differs between sexes, and perceived risk is generally higher among women than men [21]. In lieu of our findings, the strengthening of parenting support programs organized by the city currently is further recommended to address mothers' persistent concerns on risk, and potential conflicts arising from differing risk perceptions within the family. This follows the Inter-Agency Standing Committee guidelines recommending strengthening of existing resources and capacities as one of core principles in mental health support in a disaster setting [22].

\section{Empowering mothers}

PHNs, when considering mothers' distinctive concerns, perceived themselves as information channels in the community and recognized the importance of empowering residents with advice so as to allow them to make wellinformed decisions. The nurses proposed the provision and use of radiation dosimeters as an empowerment tool, and an expansion of the current scope of consultations to provide residents more opportunities to ask questions pertaining to daily life. The radiation dosimeters became widely sold commercially after the disaster, at a cost of about 30 USD. The availability and usage of radiation dosimeters is an important component for the regain of a sense of control of one's surroundings, as demonstrated by an initiative supported by the European Commission ten years after the Chernobyl accident to successfully improve mothers' sense of control by recommending the measuring of radiation levels in their living environments, and discussing protective measures that can be taken at home $[23,24]$. PHNs in Fukushima can therefore learn from these experiences and adopt similar activities in their local communities much earlier than the case of Chernobyl so as to better empower mothers and facilitate communication in radiation-related risks. Indeed, in line with recommendations by these post-Chernobyl studies [23,24], Fukushima City has already initiated experience-based learning sessions in which participating residents learn to measure and interpret their own radiation exposure level.

\section{Improving nurses' health literacy skills}

The PHNs also recognized their responsibilities and the need to improve their communication skills to better transfer scientific knowledge and information to the community. Previous research in health literacy has indicated that any information provided through parenting support and other city activities should be comprehensible to mothers and their family members [25]. Such access to information would allow residents to make informed lifestyle decisions. This might also relieve unnecessary distress in mothers and within the family. In times of crisis, high quality communication between lay people and healthcare workers is critical both in the relaying of clear and credible information and the engagement of intended audiences [26]. The National Action Plan to Improve Health Literacy has therefore been initiated in the US in 2010, with the goal of developing and disseminating health information that is accurate, accessible, and actionable [27]. Henceforth, increasing awareness of health literacy, a concept only introduced in Japan in the late 1990s [28], is an important step to further bolster quality communication between PHNs and community residents. Although no studies have been published on this front, Fukushima City and Fukushima Medical University are currently organizing and evaluating health literacy workshops for the PHNs.

\section{Expanding city health services}

Going beyond the individual capacity-building of residents and PHNs, the nurses have also, in their peer discussions, suggested an organizational upgrading of the provision of city health services to improve their communication with the community. Specific suggestions included expanding the frequency of community-based counseling services, and standardizing the provision of information (highlighting the need for systematic information especially with regards to thyroid cancer screening). The importance of improving communication between the community and the local government office, in which PHNs work, is supported by a previous study [29]. In their examination of determinants of people's trust toward government and non-government organizations, they found that an important factor to facilitate government-community relations was the degree of "openness" of an organization attained via the bidirectional communication. In general, a challenge among PHNs working in Japan's hierarchical local government system is the difficulty of initiating new activities that have not been endorsed by the upper levels of the government [30]. The disaster has however, increased the PHN's decision-making power in the local government system, thus new programs such as a mental health support program for mothers at the 18-month child health checkups, regular lectures on radiation-related topics 
for residents, and epidemiological assessments of child growth have been implemented since the Fukushima nuclear incident [10].

Although human resource and budget constraints exist, and the differing interests among stakeholders (such as upper boards of prefectural government, municipal office and universities) will need to be taken into account, continuous efforts to improve services that can enhance communication with the residents are required to address the social distrust caused in part by how the Japanese government has communicated with people immediately after the disaster [31].

\section{Study limitations and strengths}

A major limitation of the present study was the limited amount of qualitative data extracted from the child health checkup files and workshop discussion notes. Each case contained only a few sentences that were often fragments. Another limitation was how samples were only collected within a specific time period of each year (between April and May). However, the qualitative analysis of both parenting counseling records and discussion notes from PHNs' training workshops, although limited in amount and time-frame, can provide comprehensive information for PHNs and policy makers to understand community needs and to formulate appropriate strategies. Of note, a comparison between the present study and a previous study adopting a similar sampling strategy throughout a year in the sample city has indicated no differences in mothers and children's basic characteristics [32].

On the other hand, a methodological strength of this paper was that it has demonstrated the usefulness of the combination of text mining and SCAT for the analysis of text data that are readily available and accessible via currently available healthcare services.

\section{Conclusion}

The analyses of existing parenting counseling records and PHNs' peer discussions have provided a deeper understanding of the challenges pertaining to risk communication that exist in the community and the possible strategies that PHNs can act on following a disaster in Japan. These challenges include the need for mothers to be considerably informed on radiation risks as they can have impact on their relocation decisions, child safety concerns, and interpersonal conflicts within the family due to differing risk perceptions. In addition, this study has shown how PHNs perceive themselves as information channels within the community; yet they acknowledge their lack of capacity to provide information and to communicate in a way that facilitates an optimal delivery of healthcare services in a disaster setting. Our study has therefore suggested a three-tiered strategy at the resident, healthcare provider and health system levels to alleviate these challenges of risk communication: empowerment of local residents to assume more active roles in the understanding of their environment and subsequently making informed decisions about preventive measures against radiation exposure, capacity-building of PHNs in health literacy skills so as to allow them to be sources of reliable communication channels, and the expansion of health services to enhance communications between PHNs and communities. As our findings have provided a premise for the initiation of capacity-building specific to health literacy in PHNs, the Fukushima Medical University has in collaboration with some local government offices started to conduct health literacy training sessions for the PHNs. It is anticipated that the recommendations from this research will inform future efforts in disaster recovery and the strengthening of current health and social care systems during times of crisis.

\section{Competing interests}

The authors declare that they have no competing financial interests.

\section{Authors' contributions}

AG designed the study, analyzed data and wrote the manuscript. RER and AYL contributed to data analysis, interpretation and manuscript writing. KY contributed to data collection and data management. YS conceived of the study and collected data. DDH contributed to manuscript planning and writing. HK supervised the workshop and contributed to manuscript writing MRR contributed to data interpretation and manuscript writing. All authors read and approved the final manuscript.

\section{Acknowledgments}

We thank the public health nurses of Fukushima City Health and Welfare Center for their contribution in data collection, and journal reviewers for improving the manuscript. This work was supported in part by the Japan Foundation for the Promotion of International Medical Research Cooperation.

\section{Author details}

${ }^{1}$ Department of Public Health, Fukushima Medical University School of Medicine, Hikarigaoka 1, Fukushima-City, Fukushima 960-1295, Japan. ${ }^{2}$ Takemi Program in International Health, Harvard School of Public Health (at time of manuscript writing), 665 Huntington Avenue, Boston, MA 02115, USA. ${ }^{3}$ Department of Social and Behavioral Sciences, Harvard School of Public Health, 677 Huntington Avenue, Boston, MA 02115, USA. ${ }^{4}$ Department of Health Communication, School of Public Health, The University of Tokyo (at time of manuscript writing), 7-3-1 Bunkyo-ku, Hongo, Tokyo 113-8654, Japan. ${ }^{5}$ Office for Educational Programs, Harvard School of Public Health, 665 Huntington Avenue, Boston, MA 02115, USA. 'Gender-Specific Medicine Center, Fukushima Medical University School of Medicine, Hikarigaoka 1, Fukushima-city, Fukushima 960-1295, Japan. ${ }^{7}$ Department of Global Health and Population, Harvard School of Public Health, 665 Huntington Avenue, Boston, MA 02115, USA.

Received: 23 June 2013 Accepted: 6 March 2014 Published: 19 March 2014

\section{References}

1. Perko T: Importance of risk communication during and after a nuclear accident. Integr Environ Assess Manag 2011, 7:388-392.

2. The National Diet of Japan: The Fukushima Nuclear Accident Independent Investigation Commission. The official report of the Fukushima Nuclear Accident Independent Investigation Commission. Executive summary. Tokyo: The National Diet of Japan; 2012.

3. Yasumura S, Goto A, Yamazaki S, Reich MR: Excess mortality among relocated institutionalized elderly after the Fukushima nuclear disaster. Public Health 2013, 127:186-188.

4. Takahashi S, Akabayashi A: Earthquake in Japan. Lancet 2011, 377:1653-1654. 
5. McCurry J: Fukushima residents still struggling 2 years after disaster. Lancet 2013, 381(9869):791-792.

6. World Health Organization: Health risk assessment from the nuclear accident after the 2011 Great East Japan earthquake and tsunami, based on a preliminary dose estimation. Geneva: World Health Organization; 2013.

7. Apter AJ, Paasche-Orlow MK, Remillard JT, Bennett IM, Ben-Joseph EP, Batista RM, Hyde J, Rudd RE: Numeracy and communication with patients: they are counting on us. J Gen Intern Med 2008, 23:2117-2124.

8. Kitamiya C: Activities and awareness of public health nurses working at local government facilities and health centers regarding potential nuclear accidents. Japanese Journal of Public Health 2011, 58:372-381 (In Japanese).

9. Suzuki Y, Fukasawa M, Nakajima S, Narisawa T, Kim Y: Development of disaster mental health guidelines through the Delphi process in Japan. Int J Ment Health Syst 2012, 6:7.

10. Goto A: Academic institute: Parenting support strategies in areas surrounding evacuation zones. In Public Health in Nuclear Disaster. Edited by Yasumura S. Tokyo: Nanzando; 2014:333-338 (In Japanese).

11. Yoshioka $K$, Asahara $K$, Murashima S: Processes and strategies for developing public health nurse directed community health projects in municipalities of Japan: Focusing on setting agendas and making project alternatives. Japanese Journal of Public Health 2004, 51:257-271 (In Japanese).

12. Bromet EJ, Havenaar JM: Mental health consequences of disasters. In Psychiatry in Society. Edited by Sartorius N, Gaebel W, Lepez-Ibor JJ, Maj M. West Sussex: John Wiley \& Sons; 2002:241-261.

13. Higuchi $\mathrm{K}$ : Analysis of free comments in a questionnaire survey: Quantitative analysis by KH Coder. Shakai to Chosa 2012, 8:92-96 (In Japanese).

14. Chong Ho Y, Jannasch-Pennell A, Samuel DiGangi S: Compatibility between text mining and qualitative research in the perspectives of grounded theory, content analysis, and reliability. Qual Rep 2011, 16:730-744.

15. Hachiken H, Mastuoka A, Murai A, Kinoshita S, Takada M: Quantitative analyses by text mining of journal articles on medical pharmacy. Japanese Journal of Drug Informatics 2012, 13:152-159 (In Japanese).

16. Otani T: "SCAT" a qualitative data analysis method by four-step coding: Easy startable and small scale data-applicable process of theorization. Bulletin of the Graduate School of Education and Human Development (Educational Sciences) 2007-2008, 54:27-44 (In Japanese).

17. Maeno T, Takayashiki A, Anme T, Tohno E, Hara A: Japanese students' perception of their learning from an interprofessional education program: a qualitative study. Int J Med Educ 2013, 4:9-17.

18. Bromet EJ: Mental health consequences of the Chernobyl disaster. J Radiol Prot 2012, 32:N71-N75.

19. Bromet EJ, Goldgaber D, Carlson G, Panina N, Golovakha E, Gluzman SF, Gilbert T, Gluzman D, Lyubsky S, Schwartz JE: Children's well-being 11 years after the Chernobyl catastrophe. Arch Gen Psychiatry 2000, 57:563-571.

20. Tateno S, Yokoyama HM: Public anxiety, trust, and the role of mediators in communicating risk of exposure to low dose radiation after the Fukushima Daiichi Nuclear Plant explosion. JCOM 2013, 12:A03.

21. Gustafson PE: Gender differences in risk perception: theoretical and methodological perspectives. Risk Anal 1998, 18:805-811.

22. Inter-Agency Standing Committee: ISAC Guidelines on mental health and psychosocial support in emergency settings. Available at: http://www.who.int/ mental_health/emergencies/guidelines_iasc_mental_health_psychosocial_ june_2007.pdf. Accessed February 14, 2014.

23. Dubreuil GH, Lochard J, Girard P, Guyonnet JF, Le Cardinal G, Lepicard S, Livolsi P, Monroy M, Ollagnon H, Pena-Vega A, Pupin V, Rigby J, Rolevitch I, Schneider T: Chernobyl post-accident management: the ETHOS project. Health Phys 1999, 77:361-372.

24. Lochard J: Rehabilitation of living conditions in territories contaminated by the Chernobyl accident: the ETHOS project. Health Phys 2007, 93:522-526.

25. Sanders LM, Shaw JS, Guez G, Baur C, Rudd R: Health literacy and child health promotion: implications for research, clinical care, and public policy. Pediatrics 2009, 124(Suppl 3):S306-314.

26. Rudd RE, Comings JP, Hyde J: Leave no one behind: Improving health and risk communication through attention to literacy. J Health Commun 2003, 8(Suppl 1):104-115

27. U.S. Department of Health and Human Services, Office of Disease Prevention and Health Promotion: National Action Plan to Improve Health
Literacy. Available at: http://www.health.gov/communication/hlactionplan/. Accessed October 5, 2012.

28. Tokuda Y, Doba N, Butler JP, Paasche-Orlow MK Health literacy and physical and psychological wellbeing in Japanese adults. Patient Educ Couns 2009, 75:411-417.

29. Maeda Y, Miyahara M: Determinants of trust in industry, government, and citizen's groups in Japan. Risk Anal 2003, 23:303-310.

30. Japanese Nursing Association: Review of health activities in municipalities. Available at: http://www.nurse.or.jp/home/publication/pdf/senkuteki/23houkoku-shichoson.pdf. Accessed Ma 6, 2013. (In Japanese).

31. Reich MR: A public health perspective on reconstructing post-disaster Tohoku: one year later. JMAJ 2012, 55:384-387.

32. Kawai T, Goto A, Watanabe E, Nagasawa M, Yasumura S: Lower respiratory tract infections and gastrointestinal infections among mature babies in Japan. Pediatr Int 2011, 53:431-445.

doi:10.1186/1472-6963-14-129

Cite this article as: Goto et al:: Leveraging public health nurses for disaster risk communication in Fukushima City: a qualitative analysis of nurses' written records of parenting counseling and peer discussions. BMC Health Services Research 2014 14:129.

\section{Submit your next manuscript to BioMed Central and take full advantage of:}

- Convenient online submission

- Thorough peer review

- No space constraints or color figure charges

- Immediate publication on acceptance

- Inclusion in PubMed, CAS, Scopus and Google Scholar

- Research which is freely available for redistribution

Submit your manuscript at www.biomedcentral.com/submit
C Biomed Central 